\title{
Low dose of kaempferol suppresses the migration and invasion of triple-negative breast cancer cells by downregulating the activities of RhoA and Racl
}

This article was published in the following Dove Press journal:

OncoTargets and Therapy

3 October 2017

Number of times this article has been viewed

\author{
Shoushan $\mathrm{Li}^{1, *}$ \\ Ting Yan ${ }^{2, *}$ \\ Rong Deng,* \\ Xuesong Jiang ${ }^{4}$ \\ Huaping Xiong' \\ Yuan Wang' \\ Qiao $\mathrm{Yu}^{3}$ \\ Xiaohua Wang ${ }^{5}$ \\ Cheng Chen ${ }^{4}$ \\ Yichao Zhu ${ }^{6,7}$
}

'Department of Oncology, Traditional Chinese Medical Hospital of Siyang County, ${ }^{2}$ Safety Assessment and Research Center for Drug, Pesticide and Veterinary Drug of Jiangsu Province, Nanjing Medical University, ${ }^{3}$ Department of General Surgery, Jiangsu Cancer Hospital, Jiangsu Institute of Cancer Research, Nanjing Medical University Affiliated Cancer Hospital, ${ }^{4}$ Department of Radiotherapy, Jiangsu Cancer Hospital, Jiangsu Institute of Cancer Research, Nanjing Medical University Affiliated Cancer Hospital, ${ }^{5}$ Department of Chemotherapy, Jiangsu Cancer Hospital, Jiangsu Institute of Cancer Research, Nanjing Medical University Affiliated Cancer Hospital, ' ${ }^{6}$ epartment of Physiology, Nanjing Medical University, ${ }^{7}$ State Key Laboratory of Reproductive Medicine, Nanjing Medical University, Nanjing, China

*These authors contributed equally to this work

Correspondence: Xiaohua Wang Department of Chemotherapy, Jiangsu Cancer Hospital, Jiangsu Institute of Cancer Research, Nanjing Medical University Affiliated Cancer Hospital, Nanjing 210009, China

Email wangxiaohua8110@sina.com

Yichao Zhu

Department of Physiology, State Key Laboratory of Reproductive Medicine, Nanjing Medical University, Nanjing 211166, China Email zhuyichaol26@hotmail.com
Purpose: Triple-negative breast cancer (TNBC) is an especially aggressive and hard-to-treat disease. Although the anticancer role of kaempferol has been reported in breast cancer, the effect of kaempferol on TNBC remains unclear.

Materials and methods: This experiment investigated the migration-suppressive role of a low dose of kaempferol in TNBC cells. Wound-healing assays and cell invasion assays were used to confirm the migration and invasion of cells treated with kaempferol or transfected indicated constructs. We evaluated the activations of RhoA, Rac1 and Cdc42 in TNBC cells with a Rho activation assay. A panel of inhibitors of estrogen receptor/progesterone receptor/ human epidermal growth factor receptor 2 (ER/PR/HER2) treated non-TNBC (SK-BR-3 and MCF-7) cells and blocked the ER/PR/HER2 activity. Wound-healing assays and Rho activation assays were employed to measure the effect of kaempferol and ER/PR/HER2 inhibitors on Rho activation and cell migration rates.

Results: A low dose of kaempferol $(20 \mu \mathrm{mol} / \mathrm{L})$ had a potent inhibitory effect on the migration and invasion of TNBC cells, but not on the migration of non-TNBC (SK-BR-3 and MCF-7) cells. The low dose of kaempferol downregulated the activations of RhoA and Rac1 in TNBC cells. Moreover, the low dose of kaempferol also inhibited the migration and RhoA activations of HER2-silence SK-BR-3 and ER/PR-silence MCF-7 cells. Overexpressed HER2 rescued the cell migration and RhoA and Rac1 activations of kaempferol-treated MDA-MB-231 cells.

Conclusion: The low dose of kaempferol inhibits the migration and invasion of TNBC cells via blocking RhoA and Rac1 signaling pathway.

Keywords: kaempferol, TNBC, migration, invasion, RhoA, Rac1

\section{Introduction}

Triple-negative breast cancer (TNBC) is defined by a lack of estrogen receptor (ER), progesterone receptor (PR), and human epidermal growth factor receptor 2 (HER2) receptor gene copy number and expression. ${ }^{1}$ TNBC accounts for $15 \%-20 \%$ of all breast cancers and is an especially aggressive and hard-to-treat disease. ${ }^{2}$ Women with TNBC are generally diagnosed at later stages, have a poorer prognosis and a higher recurrence risk compared with those diagnosed with non-TNBC. ${ }^{3}$ TNBCs are highly heterogeneous, as cells within the tumor differ in their genetic profiles and histologies. ${ }^{4}$ The unique features of these tumors have thus far hindered researchers from discovering novel effective therapies.

Kaempferol (3,5,7-trihydroxy-2-(4-hydroxyphenyl)-4H-1-benzopyran-4-one), which is a phytoestrogen belonging to the flavonoids, is mostly found in fruits and plants. ${ }^{5}$ Kaempferol exhibits potential anti-bacterial, anti-oxidative, anti-inflammatory, 
and anti-tumor activities. ${ }^{6-9}$ Kaempferol induces growth inhibition and apoptosis in lung cancer cells by activating MEK-MAPK, and increases lung cancer cell killing by radiation in vitro and in vivo through inhibition of the AKT/ PI3K and ERK pathways and activation of the mitochondria apoptosis pathway. ${ }^{10,11}$ Kaempferol functions as an anticancer agent by re-establishing gap junctional intercellular communication through enhancement of the expression and phosphorylation of connexin 43 protein in colon cancer cells, and sensitizing colon cancer cells to tumor necrosis factor-related apoptosis -induced apoptosis. ${ }^{12,13}$ Kaempferol induces cellular apoptosis and aging through downregulating the PI3K/AKT and hTERT pathways in human cervical cancer cells. ${ }^{9}$

In breast cancer, kaempferol induces the apoptosis via cell cycle arrest, and suppresses cell growth caused by estrogen and triclosan. ${ }^{14,15}$ Kaempferol inhibits breast cancer

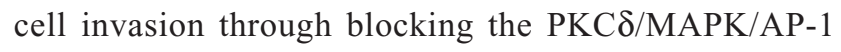
cascade and the subsequent expression and activity of matrix metalloproteinase (MMP)-9. ${ }^{16}$ Kaempferol can also suppress triclosan-induced epithelial-mesenchymal transition (EMT) and metastatic-related behaviors in MCF-7 breast cancer cells. ${ }^{17}$ Although the anticancer role of kaempferol has been successfully testified in various kinds of tumors, the effect of kaempferol on TNBC is still poorly defined. Here, we demonstrated for the first time that low dose of kaempferol suppressed the migration of TNBC cells by downregulating the Rho activity. These findings suggest that kaempferol is a potential chemotherapeutic drug for retarding TNBC metastasis.

\section{Materials and methods \\ Cell culture}

TNBC cell lines (MDA-MB-231 and MDA-MB-453), $\mathrm{ER}^{+} /$ $\mathrm{PR}^{+}$breast cancer cell line (MCF-7) and HER2 ${ }^{+}$breast cancer cell line (SK-BR-3) were purchased from the Cell Bank of Shanghai (Shanghai, China). Cells were routinely cultured in DMEM medium, supplemented with $10 \%$ fetal bovine serum (FBS; Hyclone, Logan, UT, USA), at $37^{\circ} \mathrm{C}$ in a humidified atmosphere with $5 \% \mathrm{CO}_{2}$.

\section{Transient transfections}

Breast cancer cells were seeded in 6-well plates (Costar, Corning, NY) and cultured to $80 \%$ confluence, and then transiently transfected with GFP-RhoA-V14, GFP-Rac1V12 (stored in our laboratory) or hemagglutinin (HA)-HER2 plasmids (purchased from Addgene, Cambridge, MA, USA) using Lipofectamine 2000 Reagent (Invitrogen, Carlsbad, CA, USA) in serum-free OPTI-MEM according to the manufacturer's instructions. The cells were switched to fresh medium containing 10\% FBS $6 \mathrm{~h}$ after the transfection and cultured for $48 \mathrm{~h}$. The cells transfected with GFP-RhoAV14, GFP-Rac1-V12 or HA-HER2 constructs were used for analyzing the expression and/or activation of these proteins and cell migration.

\section{Wound-healing assay}

Breast cancer cells were plated onto 96-well cell culture clusters (Costar) and grown to confluence, and then serum-starved for $24 \mathrm{~h}$. Herceptin (Genentech, South San Francisco, CA), AZD9496 (AZD; Selleck, Houston, TX, USA) or megestrol acetate (MA; Selleck) were used $60 \mathrm{~min}$ before the scratch was made. The monolayer cells were scratched manually with a plastic pipette tip, and after 2 washes with PBS, the wounded cellular monolayer was allowed to heal for $6 \mathrm{~h}$ in DMEM containing indicated dose of kaempferol (Sigma, St Louis, MO, USA). Photographs of central wound edges per condition were taken at time $0 \mathrm{~h}$ and $6 \mathrm{~h}$ using PowerShot G10 camera (Canon, Tokyo, Japan).

\section{Cell invasion assays}

Cell invasion was assessed in a modified Boyden chamber (Costar), in which the 2 chambers were separated by a polycarbonate membrane (pore diameter, $8.0 \mathrm{~mm}$ ). Boyden chamber wells were coated with matrigel (BD Biosciences, San Jose, CA, USA) for $30 \mathrm{~min}$ at $37^{\circ} \mathrm{C}$. Cells treated with indicated drugs or transfected with indicated plasmids were grown to subconfluence in tissue culture plates, then detached and thereafter centrifuged and rendered into single cell suspensions in serum-free culture medium supplemented with $5 \mathrm{mg} / \mathrm{mL}$ bovine serum albumin. The suspensions containing $5 \times 10^{4}$ cells were added to wells with a membrane placed at the bottom. The cells were allowed to migrate and invade for $6 \mathrm{~h}$ at $37^{\circ} \mathrm{C}$ in this assay. Thereafter, the medium was discarded; stationary cells were removed with a cotton-tipped applicator and the membranes were cut out of the chamber and stained with $0.5 \%$ crystal violet. The response was evaluated under a light microscope by counting the number of cells that had migrated and invaded through the membrane.

\section{Rho activation assay}

Breast cancer cells were seeded into 6-well plates and treated with indicated drugs. The experiments were then performed according to the manufacturer's protocol (Cytoskeleton Inc., Denver, CO, USA). RhoA, Cdc42 and Rac1 activation assay was performed in triplicate. The relative levels of Rho activity were normalized to the average value of controls. 


\section{Western blot analysis}

Breast cancer cells were placed into 6-well plates $\left(6 \times 10^{5}\right.$ cells/well). Seventy-two hours after the transfection with HAHER2 or vectors, the cells were harvested and homogenized with lysis buffer. Total protein was separated by denaturing $8 \%$ sodium dodecyl sulfate-polyacrylamide gel electrophoresis (SDS-PAGE). Western blot analysis was performed. ${ }^{18}$ The primary antibodies for HA and $\beta$-actin were purchased from Cell Signaling Technology (Danvers, MA, USA). Protein levels were normalized to $\beta$-actin.

\section{Pulldown assays}

For detection of active RhoA or Rac1, equal volumes of total cellular protein were incubated with GST-RBD or GST-PBD beads captured on MagneGST Glutathione Particles (Promega, Madison, WI, USA) at $4^{\circ} \mathrm{C}$ with constant rotation for $90 \mathrm{~min}$. The beads were washed 3 times with washing buffer $\left(4.2 \mathrm{mmol} / \mathrm{L} \mathrm{Na}_{2} \mathrm{HPO}_{4}, 2 \mathrm{mmol} / \mathrm{L} \mathrm{KH}_{2} \mathrm{PO}_{4}\right.$, $280 \mathrm{mmol} / \mathrm{L} \mathrm{NaCl}$, and $10 \mathrm{mmol} / \mathrm{L} \mathrm{KCl}, \mathrm{pH}$ 7.2). At the end of this period, the beads were captured by the magnet in a magnetic stand. After washing 3 times with ice-cold buffer, the beads were resuspended in Laemmli buffer, boiled, and subjected to western blot analysis. SDS-PAGE and western blot were performed using standard methods. The other procedures were described as aforementioned.

\section{Quantitative real-time PCR ( $\mathrm{PPCR}$ )}

Total RNAs were isolated with TRIzol reagent (Invitrogen). First-strand cDNAs were synthesized using total RNAs, avian myeloblastosis virus reverse transcriptase (Promega), and an oligo (dT) primer. Polymerase chain reaction (PCR) product amplification was detected by the level of fluorescence emitted by SYBR Green (SYBR ${ }^{\circledR}$ Premix Ex Taq ${ }^{\mathrm{TM}}$ II, TaKaRa Dalian, China), which intercalated into double-stranded DNA. Primers used for PCR amplification were as follows: Vimentin forward, 5'-CAGATGCGTGAAATGGAAGA-3', Vimentin reverse, 5'-CTCAATGTCAAGGGCCATCT-3'; E-cadherin forward, 5'-CAGCACGTACACAGCCC TAA-3', E-cadherin reverse, 5'-TGAGGCTTTG GATTCCTCTC-3'; Snail forward, 5'-CCTCCACGA GGTGTGACTAACT-3', Snail reverse, 5'-CCGACAAG TGACAGCCATTA-3'; Slug forward, 5'-CGCAATCA ATGTTTACTCGAAC-3', Slug reverse, 5'-TCTCAAT CTAGCCATCAGCAAA-3'; MMP2 forward, 5'-AGAC ATACATCTTTGCTGGAG-3', MMP2 reverse, 5'-ATC TGCGATGAGCTTGG-3'; MMP9 forward, 5'-CC ACTGCTGGCCCTTCTA-3', MMP9 reverse, 5'-CCCT GCCCTCAGAGAAT-3'; GAPDH forward, 5'-GCTG
CGAAGTGGAAACCATC-3', GAPDH reverse, 5'-CCT CCTTCTGCACACATTTGAA- $3^{\prime}$. The $\Delta \mathrm{Ct}$ method was used for mRNA expression analysis. First, the cycle number at the threshold level of fluorescence $(\mathrm{Ct})$ for each sample was determined. Next, the $\Delta \mathrm{Ct}$ value was calculated. The $\Delta \mathrm{Ct}$ value was the difference between the $\mathrm{Ct}$ value of indicated genes and the $\mathrm{Ct}$ value of glyceraldehyde 3-phosphate dehydrogenase (GAPDH): $\Delta \mathrm{Ct}=\mathrm{Ct}$ (indicated gene) $-\mathrm{Ct}$ (GAPDH). The fold-change for mRNA from cells relative to each control cells was calculated using the $2^{-\Delta \Delta \mathrm{Ct}}$ method. PCR was performed in triplicate.

\section{Statistical analysis}

Each experiment was repeated for at least three times. Numerical data were presented as mean \pm SD. The difference between means was analyzed with Student's $t$-test. All statistical analyses were performed using SPSS 13.0 software (SPSS Inc., Chicago, IL, USA). Differences were considered significant when $P<0.05$.

\section{Results \\ Low dose of kaempferol inhibits the migration of TNBC cells}

Previous studies reported that kaempferol inhibits cell invasion and EMT of MDA-MB-231 and MCF-7 breast cancer cells. ${ }^{16,17}$ To explore whether low dose of kaempferol specifically suppresses the migration of TNBC cells, we treated TNBC MDA-MB-231 and MDA-MB-453 cells with indicated low dose of kaempferol, and measured the migration rate. The low dose of kaempferol $(10,20,40 \mu \mathrm{mol} / \mathrm{L})$ significantly inhibited the migration of MDA-MB-231 and MDA-MB453 cells (Figure $1 \mathrm{~A}-\mathrm{C}$ ). Thus, kaempferol $20 \mu \mathrm{mol} / \mathrm{L}$ was used for further studies to identify the mechanism whereby changes in the migration of TNBC cells were induced. Next, we accessed the effect of low dose of kaempferol on nonTNBC cells. The low dose of kaempferol $(20$ and $40 \mu \mathrm{mol} / \mathrm{L})$ did not inhibit the migration of MCF-7 ER $/ \mathrm{PR}^{+}$breast cancer cells and SK-BR-3 HER2 ${ }^{+}$breast cancer cells (Figure 1D and E). However, the high dose of kaempferol $(200 \mu \mathrm{mol} / \mathrm{L})$ significantly inhibited the migration of MCF-7 and SK-BR-3 HER $2^{+}$cells (Figure 1D and E).

We also measured the invasive rate of TNBC cells treated with low dose of kaempferol. We found that the low dose of kaempferol $(20 \mu \mathrm{mol} / \mathrm{L})$ significantly retarded the invasion of MDA-MB-231 and MDA-MB-453 cells (Figure $1 \mathrm{~F}-\mathrm{H}$ ). Taken together, these results show that the low dose of kaempferol inhibits the migration and invasion of TNBC cells. 
A
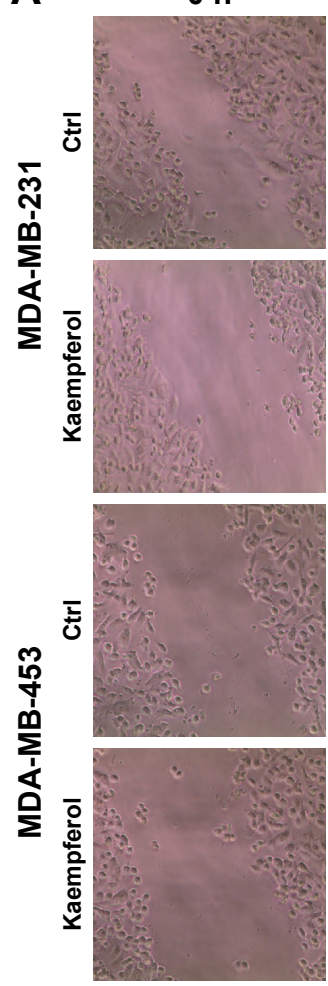

$6 \mathrm{~h}$
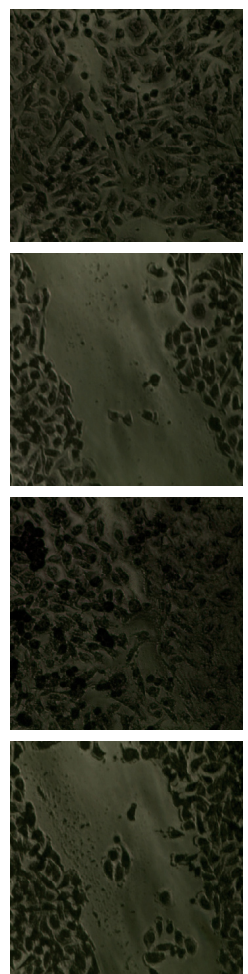

MCF-7

D

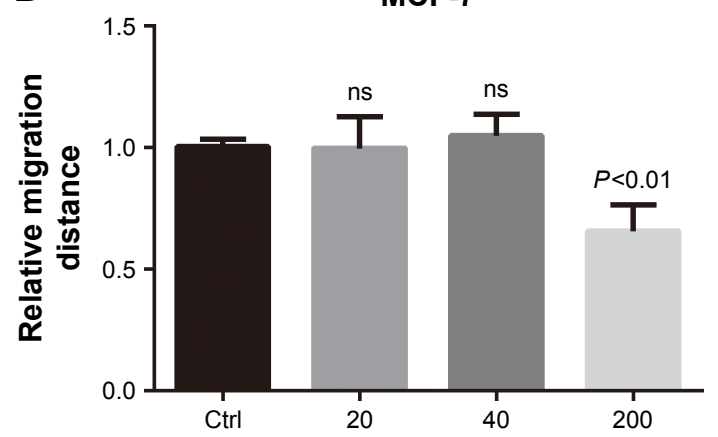

B

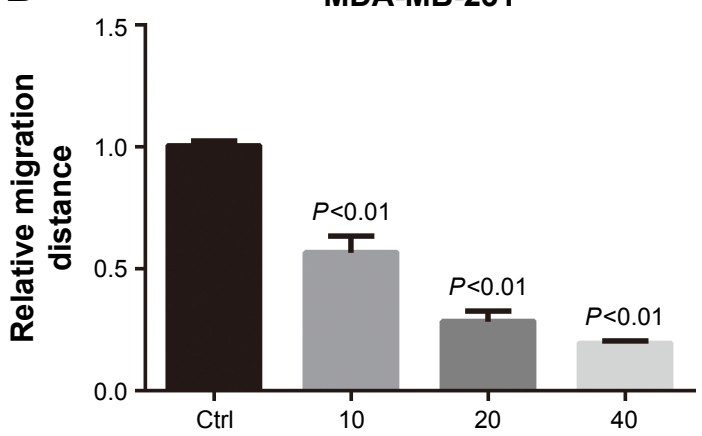

C MDA-MB-453

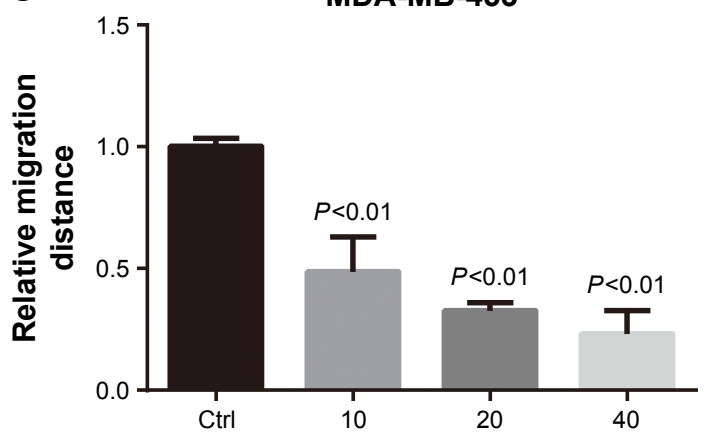

E SK-BR-3

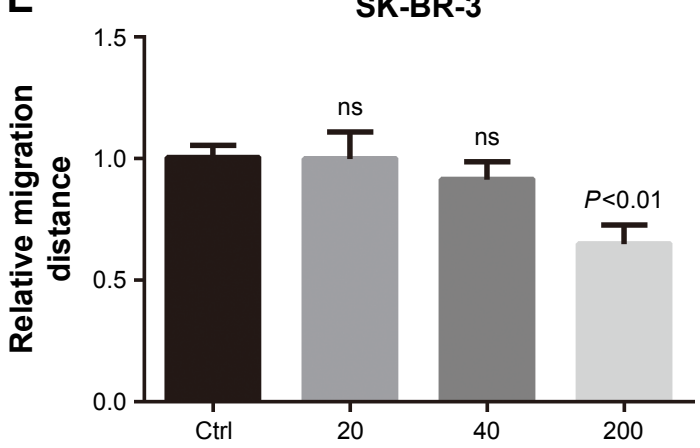

$\mathbf{F}$

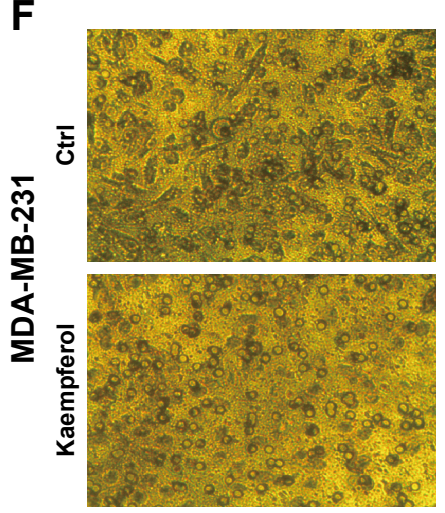

G

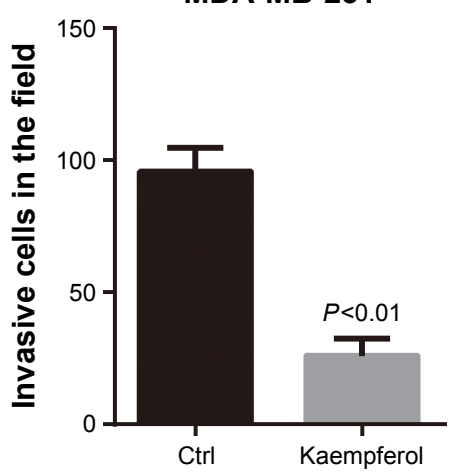

H
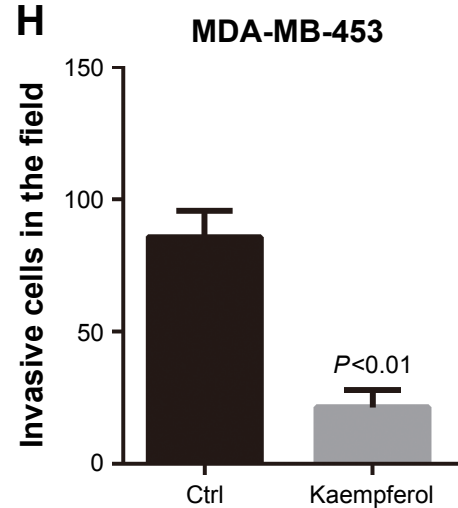

Figure I Kaempferol inhibits the migration and invasion of TNBC cells.

Notes: Breast cancer cells were incubated with kaempferol at the indicated doses for $6 \mathrm{~h}$. The cell motility rate was measured by wound healing assays. (A) Representative pictures of wound healing assays. Magnification $\times 100$. (B, C) MDA-MB-23I and MDA-MB-453 TNBC cells were allowed to migrate in response to indicated doses of kaempferol $(\mu \mathrm{mol} / \mathrm{L})$ for $6 \mathrm{~h}$. (D) MCF-7 ER $/ \mathrm{PR}^{+}$breast cancer cells were allowed to migrate in response to indicated doses of kaempferol ( $\mu \mathrm{mol} / \mathrm{L}$ ) for $6 \mathrm{~h}$. (E) SK-BR-3 HER2 ${ }^{+}$breast cancer cells were allowed to migrate in response to indicated doses of kaempferol ( $\mu \mathrm{mol} / \mathrm{L}$ ) for $6 \mathrm{~h}$. Results are presented as mean \pm SD of 5 independent experiments in (B-E). (F) The representative pictures of cell invasion assays. Magnification $\times 100$. (G, H) MDA-MB-23I and MDA-MB-453 TNBC cells were allowed to invade in response to $20 \mu \mathrm{mol} / \mathrm{L}$ kaempferol for $6 \mathrm{~h}$. Results are presented as mean \pm SD of 3 independent experiments in $(\mathbf{G}$ and $\mathbf{H})$.

Abbreviations: Ctrl, control; ER, estrogen receptor; HER2, human epidermal growth factor receptor 2; PR, progesterone receptor; TNBC, triple-negative breast cancer; ns, no significance. 


\section{Kaempferol inhibits cell migration and invasion by downregulating RhoA and Racl activations}

In view of the fact that Rho signaling mediates microfilament rearrangement and cell migration of cancer cells, ${ }^{18-22}$ we speculated that kaempferol inhibited the migration of TNBC cells by downregulating Rho signaling. We treated TNBC cells with low dose of kaempferol, and then examined the activation of RhoA, Rac1 and Cdc42 by Rho activation assays and Pull-down assays. As a result, the activations of RhoA and Rac1 were significantly blocked by kaempferol treatment in MDA-MB-231 and MDA-MB-453 cells (Figure 2A-D). However, Cdc42 activation was not altered in kaempferol-treated MDA-MB-231 and MDA-MB-453 cells (Figure 2A and B).

We next transfected TNBC cells with constitutive activity RhoA (RhoA-V14) and Rac1 (Rac1-V12), and then determined the migration and invasion rates. The transfection efficiency of GFP-tagged puncta were $60 \%-70 \%$, as observed under a fluorescence microscope. Overexpression of RhoA-V14 and Rac1-V12 elevated the RhoA and Rac1 activations of kaempferol-treated MDA-MB-231 and
MDA-MB-453 cells, respectively (Figure 3A and D). Moreover, the constitutive activity RhoA and Racl rescued the migration and invasion of kaempferol-treated TNBC cells (Figure 3B, C, E, and F). Collectively, the low dose of kaempferol inhibits cell migration and invasion by downregulating RhoA and Rac1 activations in TNBC cells.

\section{Kaempferol and herceptin suppress the migration and Rho activity of HER2 ${ }^{+}$cells}

Having identified its role as a migration-suppressor on TNBC cells, we further determined the effect of kaempferol on HER2 ${ }^{+}$breast cancer cells that were treated with herceptin. The low dose of kaempferol $(20 \mu \mathrm{mol} / \mathrm{L})$ significantly inhibited the migration of HER2-silence SK-BR3 cells (Figure 4A). We also found that the activations of RhoA and Rac1 were down-regulated in HER2-silence SK-BR3 cells (Figure 4B and C).

\section{Overexpression of HER2 rescues the migration and invasion, and Rho activity of kaempferol-treated TNBC cells}

We transfected the HA-tagged HER2 into MDA-MB-231 TNBC cells, then examined the HER2 expression by using
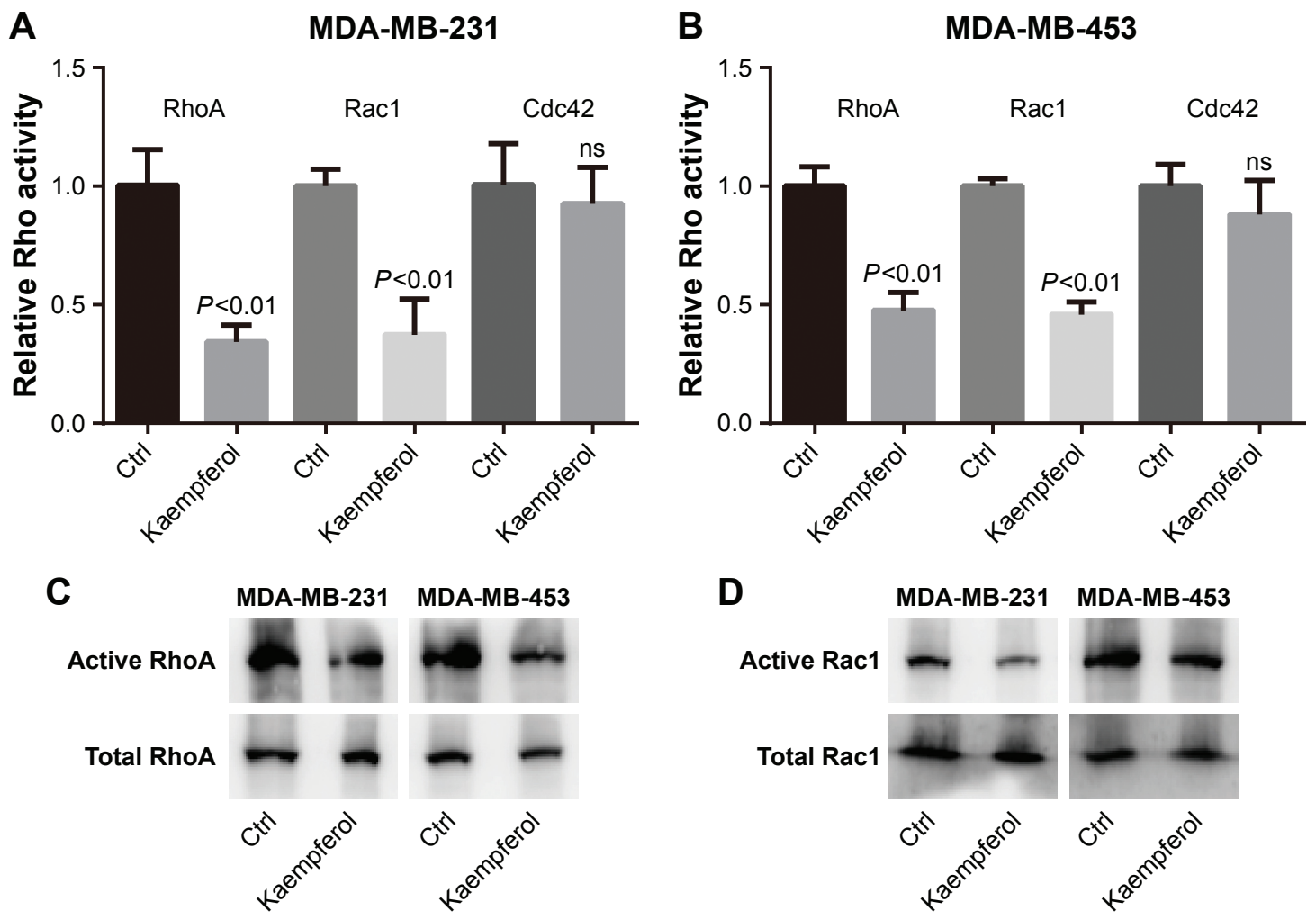

Figure 2 Kaempferol depresses the activations of RhoA and Racl in TNBC cells.

Notes: (A, B) TNBC cells (MDA-MB-23I and MDA-MB-453) were incubated with $20 \mu \mathrm{mol} / \mathrm{L}$ kaempferol for I h, and then subjected to the Rho activity assays. The relative levels of Rho activity were normalized to the average value of controls. Results are presented as mean \pm SD of 3 independent experiments. (C, D) TNBC cells (MDA-MB-23I and MDA-MB-453) were incubated with $20 \mu \mathrm{mol} / \mathrm{L}$ kaempferol for I h, and then subjected to the pulldown assays and Western blot analysis.

Abbreviations: Ctrl, control; ns, no significance; TNBC, triple-negative breast cancer. 
A

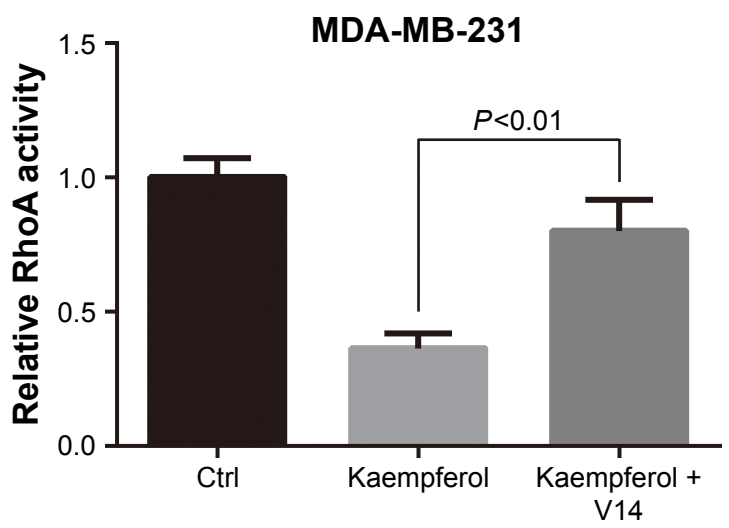

C

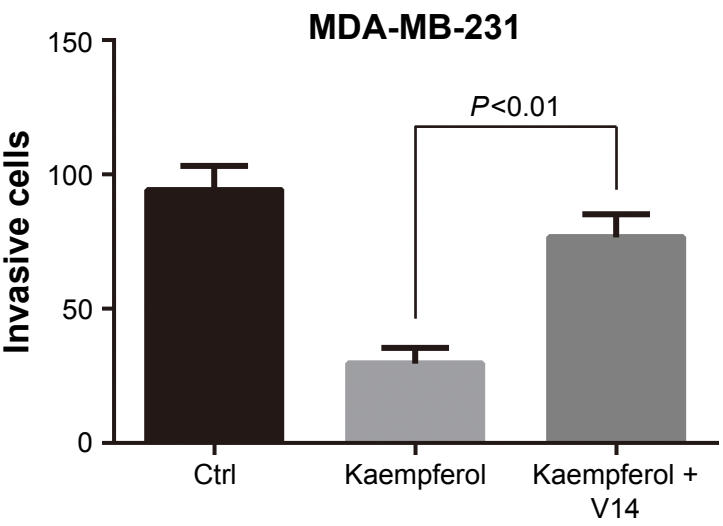

$\mathbf{E}$

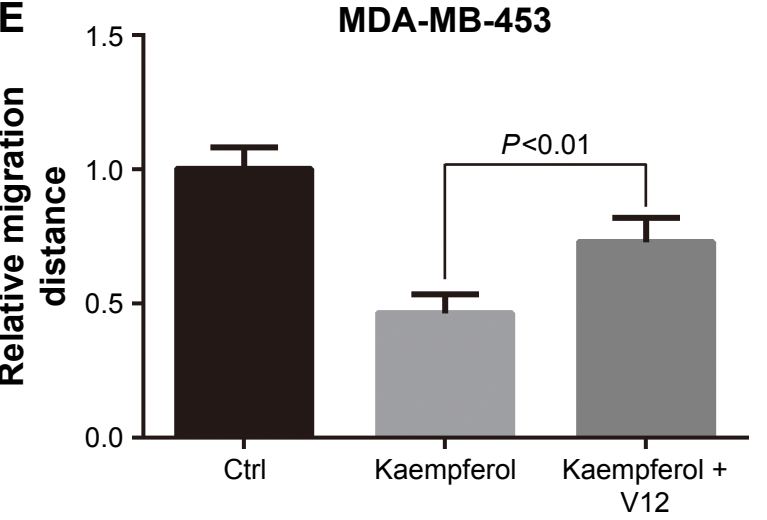

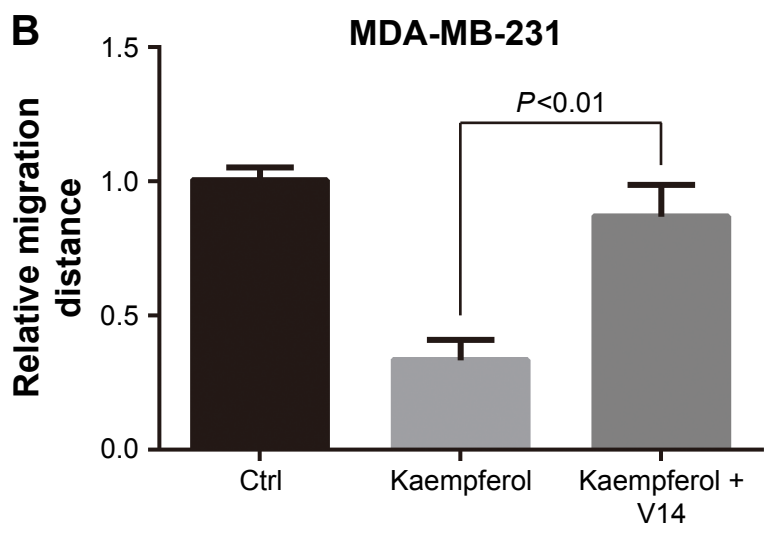

D

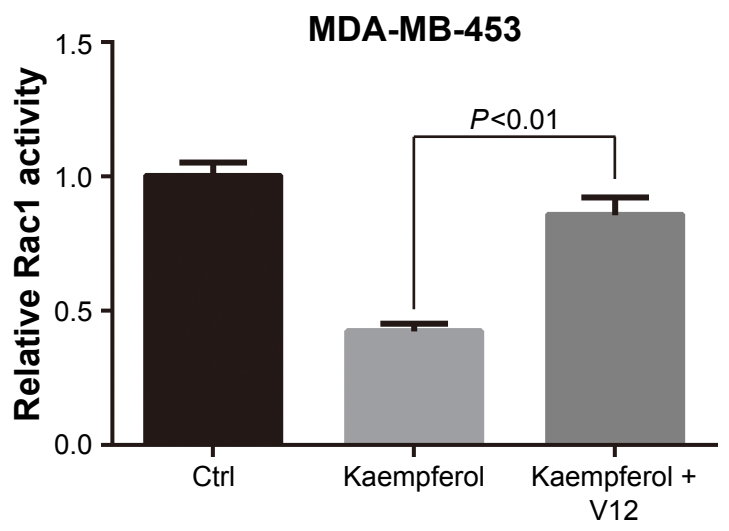

$\mathbf{F}$

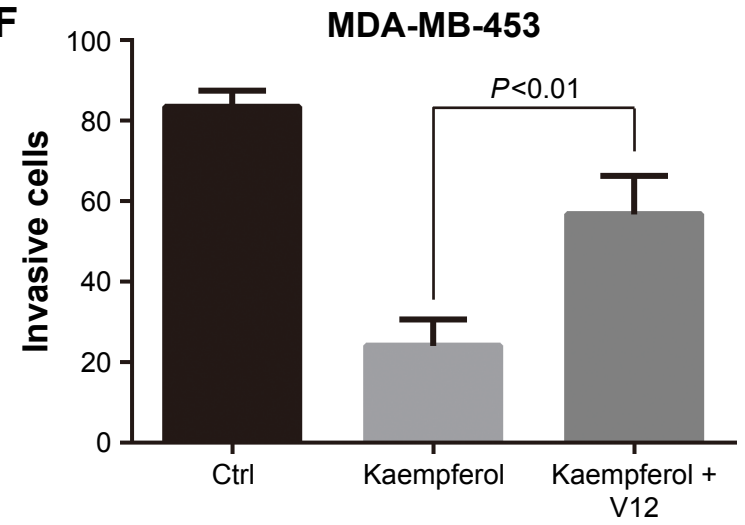

Figure 3 Constitutive activity RhoA and Racl rescue the migration and invasion of TNBC cells inhibited by kaempferol.

Notes: (A-C) MDA-MB-23I TNBC cells were transfected with constitutive activity RhoA (RhoA-VI4) or vector, then subjected to the RhoA activity assays (A), wound healing assays (B), and cell invasion assays (C). (D-F) MDA-MB-453 TNBC cells were transfected with constitutive activity Racl (Racl-VI2) or vector, then subjected to the Racl activity assays (D), wound healing assays (E) and cell invasion assays (F). The relative levels of Rho activity and cell migration distance were normalized to the average value of controls. Results are presented as mean \pm SD of 3 independent experiments in (A, C, D, F) and 5 independent experiments in (B, E).

Abbreviations: Ctrl, control; TNBC, triple-negative breast cancer.

western blot assays. HER2 were extremely expressed in HA-HER2-transfected MDA-MB-231 cells (Figure 5A). In those cells, the low dose of kaempferol $(20 \mu \mathrm{mol} / \mathrm{L})$ did not inhibit the cell migration, the activation of RhoA and Rac1, and the cell invasion (Figure 5B-E). Thus, the overexpression of HER 2 could rescue the migration and invasion, and Rho activity of kaempferol-treated TNBC cells.

\section{Kaempferol, AZD and MA suppress} the migration and Rho activity of $\mathrm{ER}^{+} / \mathrm{PR}^{+}$cells

We further determined the kaempferol's effect on $\mathrm{ER}^{+} /$ $\mathrm{PR}^{+}$breast cancer cells that were treated with AZD and MA. The low dose of kaempferol $(20 \mu \mathrm{mol} / \mathrm{L})$ significantly inhibited the migration of ER/PR-silence 

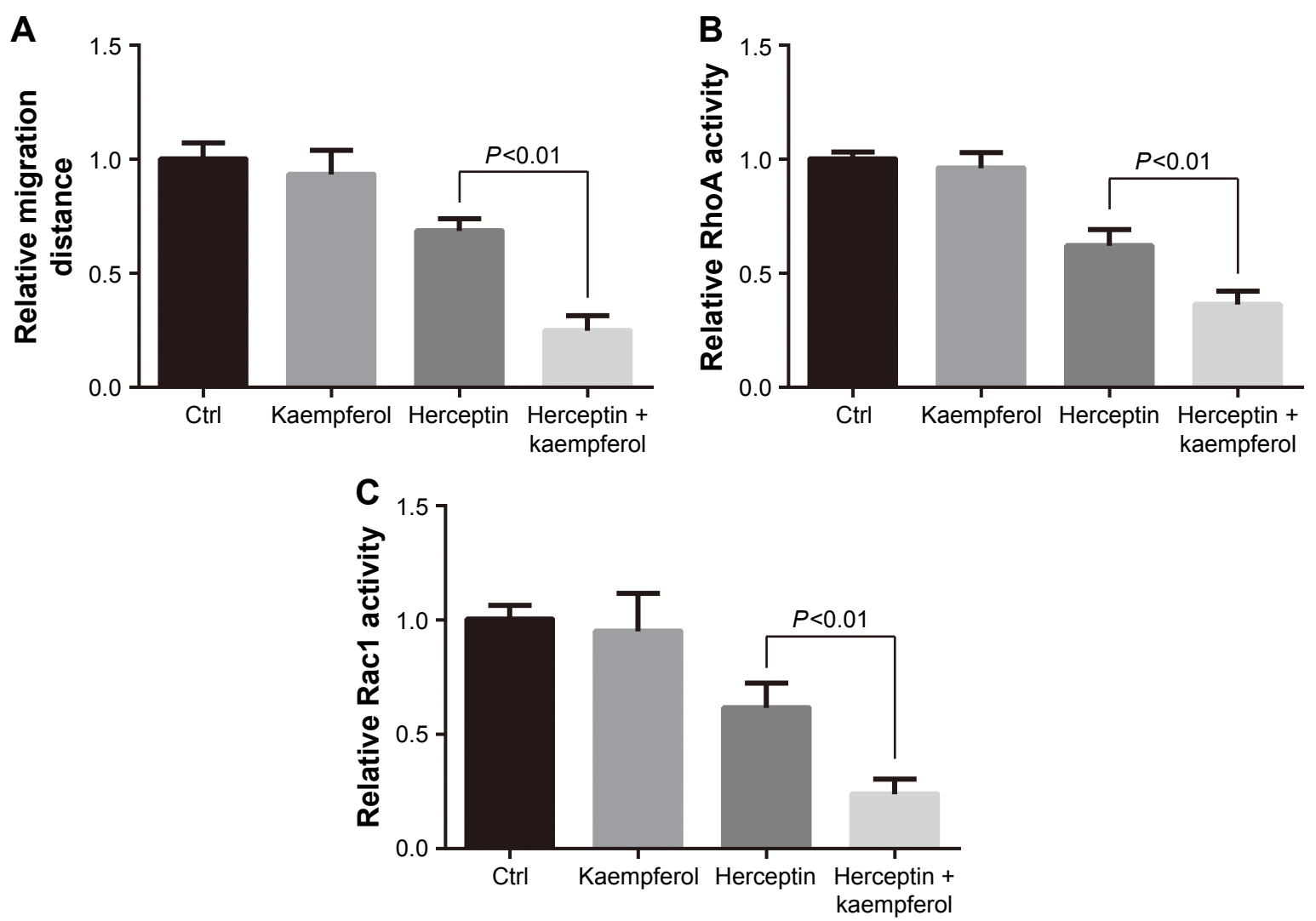

Figure 4 Kaempferol and HER2 inhibitor suppress the migration and Rho activity of SK-BR-3 cells.

Notes: (A) SK-BR-3 HER2 ${ }^{+}$breast cancer cells were allowed to migrate in response to $20 \mu \mathrm{mol} / \mathrm{L}$ kaempferol and/or $10 \mu \mathrm{g} / \mathrm{mL}$ herceptin (HER2 inhibitor) for 6 h. Results are presented as mean \pm SD of 5 independent experiments. (B, C) SK-BR-3 cells were incubated with $20 \mu \mathrm{mol} / \mathrm{L}$ kaempferol and/or I0 $\mu \mathrm{g} / \mathrm{mL}$ herceptin for I h, and then subjected to the RhoA (B) and Racl (C) activity assays. The relative levels of Rho activity were normalized to the average value of controls. Results are presented as mean \pm SD of 3 independent experiments.

Abbreviations: Ctrl, control; HER2, human epidermal growth factor receptor 2.

MCF-7 cells (Figure 6A). We also found that the activation of RhoA was down-regulated in ER/PR-silenced MCF-7 cells (Figure 6B). However, the active Rac1 was not altered in ER/PR-silenced MCF-7 cells (Figure 6C).

\section{Kaempferol down-regulates the mRNA levels of EMT markers and MMPs in TNBC cells}

Finally, we checked the effect of kaempferol on the transcriptional levels of EMT markers and MMPs. The expression levels of E-cadherin (the epithelial marker) were down-regulated in MDA-MB-231 TNBC cells treated with $20 \mu \mathrm{mol} / \mathrm{L}$ kaempferol compared with those in control cells (Figure 7A). On the contrary, the expression levels of Vimentin (the mesenchymal marker), Snail and Slug were upregulated in MDA-MB-231 cells treated with kaempferol compared with those in control cells (Figure 7A). Moreover, we found that the expression levels of MMP2 and MMP9 were down-regulated in MDA-MB-231 cells treated with kaempferol compared with those in control cells (Figure 7B).

\section{Discussion}

Clinically important therapeutic advances in the treatment of TNBC have not occurred. Distant metastatic recurrences tend to occur within the first 3-5 years after the diagnosis of TNBC. ${ }^{1}$ The survival duration of patients with metastatic TNBC is notably shorter than that of patients with metastatic ER-positive cancers. ${ }^{23}$ The high mortality of TNBC has prompted researchers to investigate novel drugs targeting TNBC cells.

Kaempferol, a phytoestrogen belonging to the flavonoids, exhibits potential and anti-tumor effect. ${ }^{5}$ Kaempferol induces the apoptosis and inhibits the cell proliferation of breast cancer cells. ${ }^{14,24,25}$ Kaempferol could also inhibit breast cancer cell invasion and suppress triclosan-induced EMT and metastaticrelated behaviors. ${ }^{16,17}$ However, the effect of kaempferol on TNBC is poorly systematically defined. Here, we found that 


\section{A}
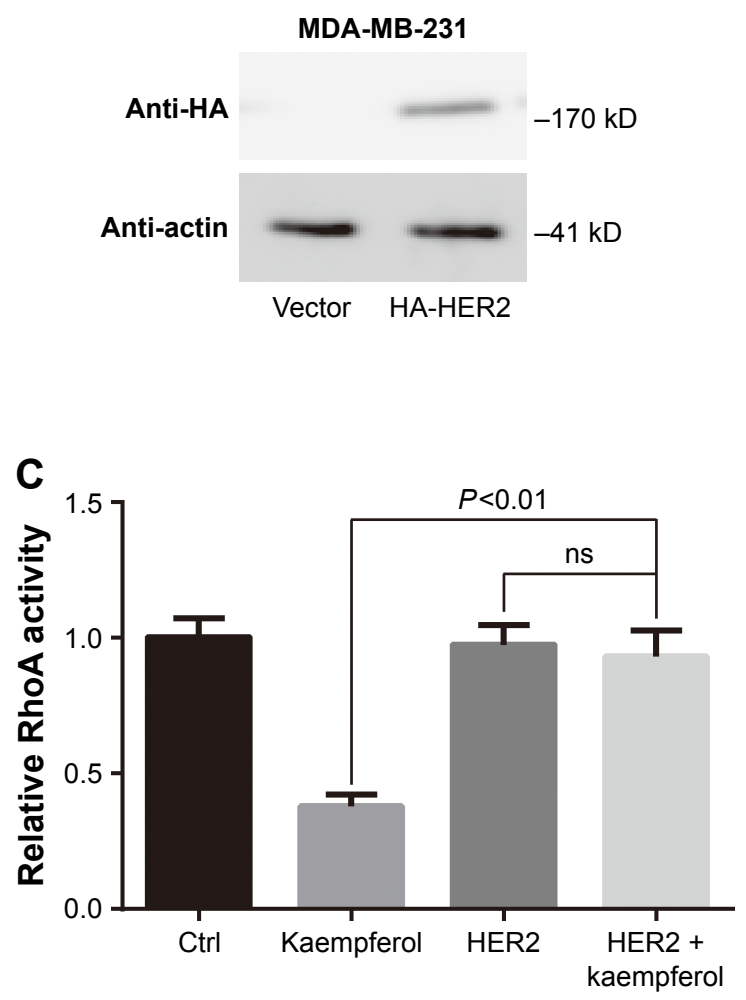

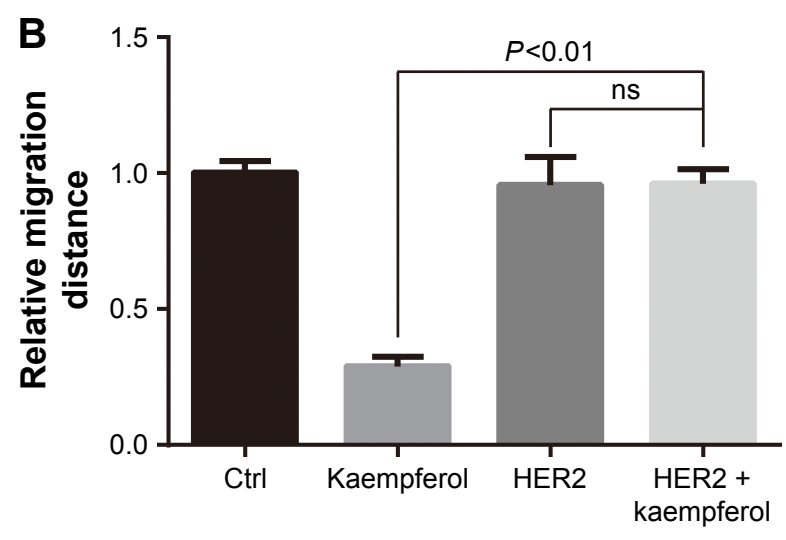

D

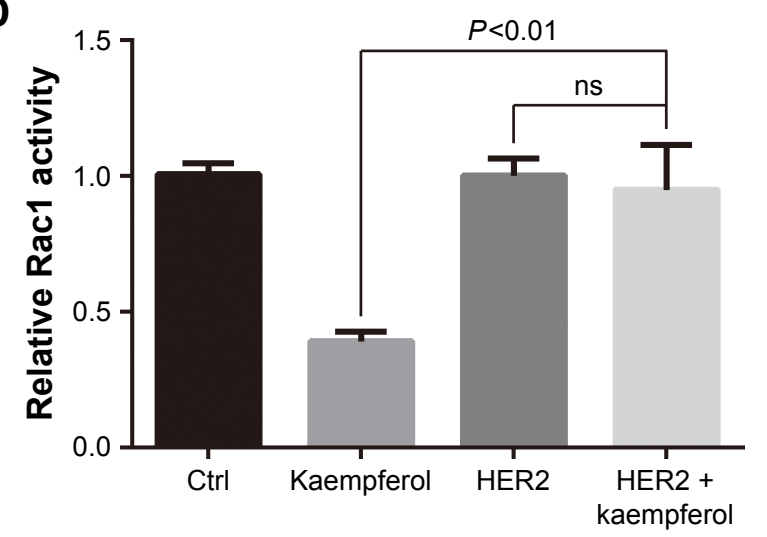

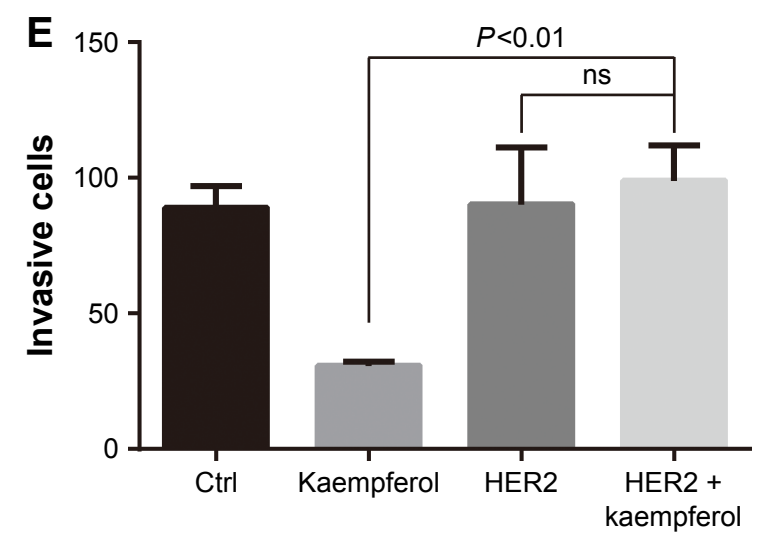

Figure 5 Overexpression of HER2 rescues the migration and Rho activity of TNBC cells inhibited by kaempferol.

Notes: (A) MDA-MB-23I TNBC cells were transfected with HA-HER2 or vector, and then subjected to Western blot analysis. $\beta$-actin as the internal control. (B) MDAMB-23I TNBC cells were transfected with HA-HER2 or vector, then allowed to migrate in response to $20 \mu$ mol/L kaempferol for $6 \mathrm{~h}$. Results are presented as mean \pm SD of 5 independent experiments. (C, D) MDA-MB-23I TNBC cells were transfected with HA-HER2 or vector, then incubated with $20 \mu$ mol/L kaempferol for I h, and subsequently subjected to the RhoA (C) and Racl (D) activity assays. The relative levels of Rho activity were normalized to the average value of controls. Results are presented as mean \pm SD of 3 independent experiments. (E) MDA-MB-23I TNBC cells were transfected with HA-HER2 or vector, then allowed to invade in response to $20 \mu \mathrm{mol} / \mathrm{L}$ kaempferol for $6 \mathrm{~h}$. Results are presented as mean \pm SD of 3 independent experiments.

Abbreviations: Ctrl, control; HER2, human epidermal growth factor receptor 2; ns, no significance; TNBC, triple-negative breast cancer.

the low dose of kaempferol effectively retards the migration of TNBC cells, but remains unavailable in non-TNBC cells' motion. Moreover, the low dose of kaempferol also inhibits the migration of HER2-silence SK-BR-3 and ER/PR-silence MCF-7 cells. Overexpressed HER2 rescues the cell migration of kaempferol-treated MDA-MB-231 cells. Thus, we declaim the role of tumor migration and invasion inhibition for the low dose of kaempferol via blocking a novel signaling pathway rather than ER/PR/HER2 signaling.

Herceptin is the prototype HER2-directed therapy that showed efficacy in early stage disease. ${ }^{26}$ Despite the practicechanging impact of herceptin and improvement in outcomes of women with HER2-positive breast cancer, resistance to herceptin is a major clinical issue. ${ }^{26}$ Here, we found that the 
A

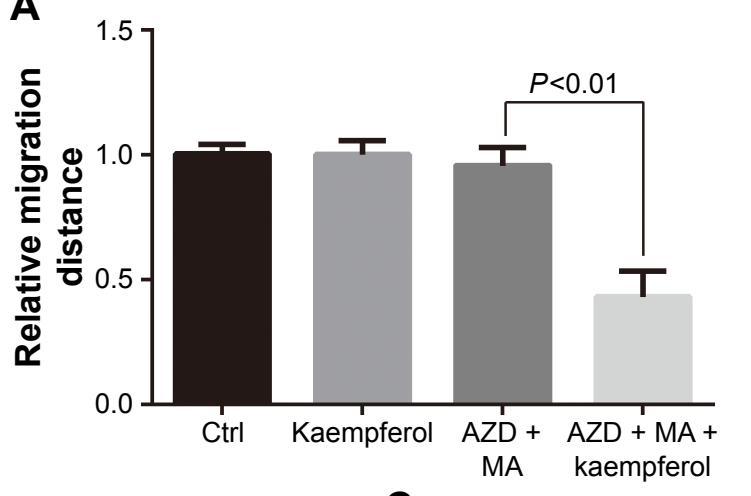

B

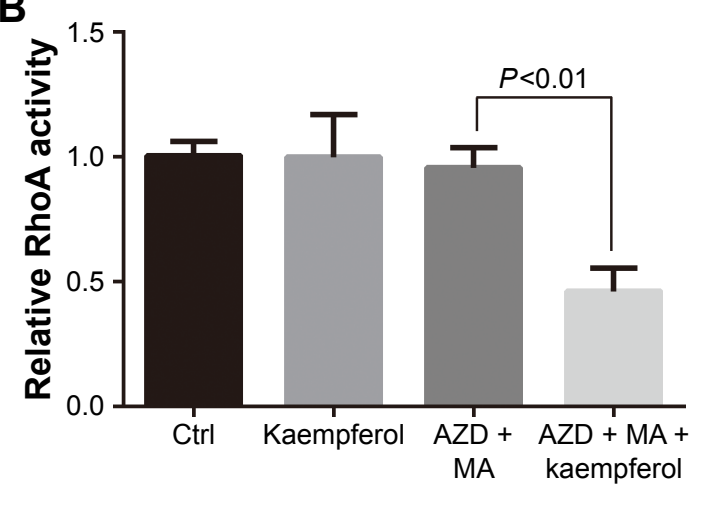

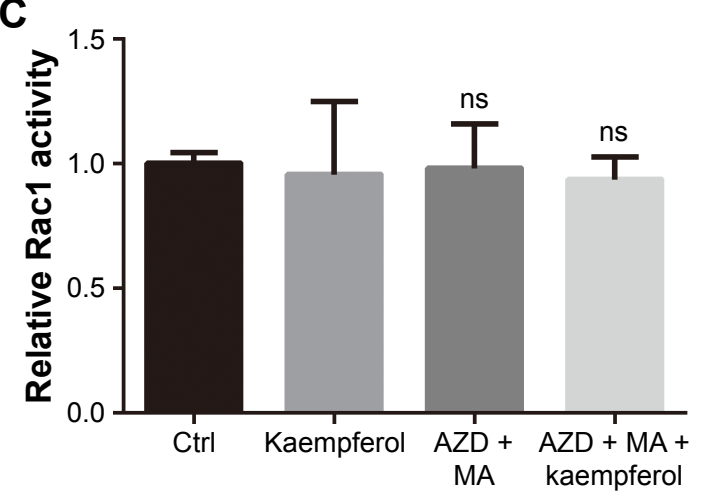

Figure 6 Kaempferol and ER/PR inhibitor suppress the migration and Rho activity of MCF-7 cells.

Notes: (A) MCF-7 ER $/ \mathrm{PR}^{+}$breast cancer cells were allowed to migrate in response to $20 \mu \mathrm{mol} / \mathrm{L}$ kaempferol and/or $0.1 \mathrm{nmol} / \mathrm{L}$ AZD9496 (ER inhibitor) and $200 \mu \mathrm{mol} / \mathrm{L}$ megestrol acetate (PR inhibitor) for $6 \mathrm{~h}$. Results are presented as mean \pm SD of 5 independent experiments. (B, C) MCF-7 cells were incubated with $20 \mu$ mol/L kaempferol and/or $0.1 \mathrm{nmol} / \mathrm{L}$ AZD9496 and $200 \mu \mathrm{mol} / \mathrm{L}$ MA for I h, and then subjected to the RhoA (B) and Racl (C) activity assays. The relative levels of Rho activity were normalized to the average value of controls. Results are presented as mean \pm SD of 3 independent experiments.

Abbreviations: AZD, AZD9496; Ctrl, control; ER, estrogen receptor; MA, megestrol acetate; ns, no significance; PR, progesterone receptor.

combination of the low dose of kaempferol with herceptin deeply inhibits the migration of HER2 ${ }^{+}$breast cancer cells. Moreover, the combination of the low dose of kaempferol with $\mathrm{AZD}$ and MA significantly blocks the migration of $\mathrm{ER}^{+} / \mathrm{PR}^{+}$breast cancer cells. These results indicate that kaempferol functions as a modulator of ER/PR and HER2 on sensitivity of breast cancer cells.

Kaempferol is poorly absorbed, with an extremely poor oral bioavailability. ${ }^{27}$ However, it has been reported that the combination of kaempferol with quercetin significantly
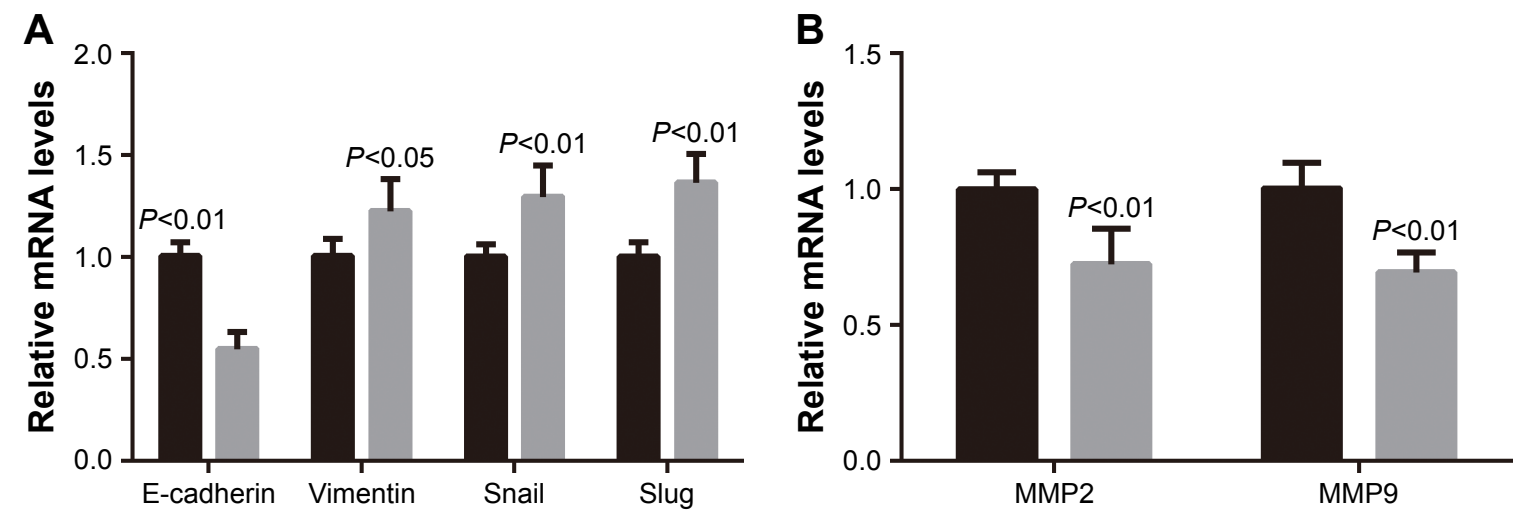

\section{Ctrl Kaempferol}

Figure 7 Kaempferol downregulates the mRNA levels of EMT markers and MMPs in TNBC cells.

Notes: (A, B) MDA-MB-23I TNBC cells were incubated with $20 \mu \mathrm{mol} / \mathrm{L}$ kaempferol for I $\mathrm{h}$, and then subjected to the qPCR assays. The relative mRNA levels of E-cadherin, Vimentin, Snail, Slug (A), and MMP2, MMP9 (B) were normalized to the average value of controls. Results are presented as mean \pm SD of 3 independent experiments. Abbreviations: EMT, epithelial-mesenchymal transition; MMP, matrix metalloproteinase; qRT-PCR, quantitative real-time polymerase chain reaction; TNBC, triple-negative breast cancer. 
increases the anticancer effects of quercetin through blocking the efflux of quercetin. ${ }^{28}$ The consumption of kaempferol has been reported to significantly increase the cytotoxic effects of cisplatin. ${ }^{29}$ In in vivo xenografted mouse models, kaempferol inhibits the growth and metastasis of cholangiocarcinoma in vivo. ${ }^{30}$ The volume of subcutaneous xenograft in the kaempferol-treated group was smaller than that in the cholangiocarcinoma control group. ${ }^{30}$ Kaempferol also suppressed the number and volume of metastasis foci in the lung metastasis model. ${ }^{30}$ Song et al also found that kaempferol, administered $20 \mathrm{mg} / \mathrm{kg} /$ day intraperitoneal injection (i.p.) daily for 3 weeks, could inhibit the growth of the gastric tumor xenografts. ${ }^{31} 17 \beta$-estradiol (E2) or triclosan-induced breast tumor growth was inhibited by co-treatment with kaempferol. ${ }^{15}$ These literatures on the anti-tumor effects of kaempferol provide the in vivo evidences supporting our findings obtained from cellular levels.

Our group has reported that Rho signaling mediates microfilament rearrangement and cell migration of cancer cells. ${ }^{18-22}$ Kaempferol blocks the PKC $\delta / M A P K / A P-1$ cascade and the subsequent expression and activity of MMP-9, and then inhibits cell invasion of breast cancer. ${ }^{16}$ Here, we found that the activations of RhoA and Rac1 were downregulated in kaempferol-treated TNBC cells. Moreover, the low dose of kaempferol also inhibits the Rho activity of HER2-silence SK-BR-3 and ER/PR-silence MCF-7 cells. Overexpressed HER2 rescues the Rho activity of kaempferol-treated MDA-MB-231 cells. Therefore, the low dose of kaempferol suppresses the migration of TNBC cells by downregulating the activities of RhoA and Rac1.

\section{Conclusion}

This study clarified the anti-tumor effect of the low dose of kaempferol on TNBC cells. To the best of our knowledge, this is the first to demonstrate that the low dose of kaempferol inhibits the migration and invasion of TNBC cells via targeting RhoA or Rac1 signaling pathway. Therefore, kaempferol is a potential therapeutic drug for human TNBC.

\section{Acknowledgments}

This work was supported by a grant from the National Natural Science Foundation of China (81472703) to Yichao Zhu, a sponsorship of Jiangsu Overseas Research and Training Program for University Prominent Young and Middle-aged Teachers and Presidents to Yichao Zhu, a grant from the Science and Technology Bureau of Suqian to Shoushan Li (Z201731), and a grant from Joint Research Project of Southeast University and Nanjing Medical University (2242017K3DN41) to Yichao Zhu.

\section{Disclosure}

The authors report no conflicts of interest in this work.

\section{References}

1. Szekely B, Silber AL, Pusztai L. New therapeutic strategies for triple-negative breast cancer. Oncology (Williston Park). 2017;31(2): 130-137.

2. Agarwal G, Nanda G, Lal P, et al. Outcomes of triple-negative breast cancers (TNBC) compared with non-TNBC: does the survival vary for all stages? World J Surg. 2016;40(6):1362-1372.

3. Dietze EC, Sistrunk C, Miranda-Carboni G, O’Regan R, Seewaldt VL. Triple-negative breast cancer in African-American women: disparities versus biology. Nat Rev Cancer. 2015;15(4):248-254.

4. Bursts of chromosome changes drive TNBC. Cancer Discov. 2016; 6(10):1075.

5. Rao KV, Seshadri TR. Synthesis of the monomethyl ethers of kaempferol, and the constitution of rhamnocitrin. J Chem Soc. 1947: 122-124.

6. Li XM, Luo XG, Si CL, et al. Antibacterial active compounds from hypericum ascyron L. induce bacterial cell death through apoptosis pathway. Eur J Med Chem. 2015;96:436-444.

7. Jing P, Song LH, Shen SQ, Zhao SJ, Pang J, Qian BJ. Characterization of phytochemicals and antioxidant activities of red radish brines during lactic acid fermentation. Molecules. 2014;19(7):9675-9688.

8. Tang XL, Liu JX, Dong W, et al. Protective effect of kaempferol on LPS plus ATP-induced inflammatory response in cardiac fibroblasts. Inflammation. 2015;38(1):94-101.

9. Kashafi E, Moradzadeh M, Mohamadkhani A, Erfanian S. Kaempferol increases apoptosis in human cervical cancer HeLa cells via PI3K/ AKT and telomerase pathways. Biomed Pharmacother. 2017;89: 573-577.

10. Nguyen TT, Tran E, Ong CK, et al. Kaempferol-induced growth inhibition and apoptosis in A549 lung cancer cells is mediated by activation of MEK-MAPK. J Cell Physiol. 2003;197(1):110-121.

11. Kuo WT, Tsai YC, Wu HC, et al. Radiosensitization of non-small cell lung cancer by kaempferol. Oncol Rep. 2015;34(5):2351-2356.

12. Nakamura Y, Chang CC, Mori T, et al. Augmentation of differentiation and gap junction function by kaempferol in partially differentiated colon cancer cells. Carcinogenesis. 2005;26(3):665-671.

13. Yoshida T, Konishi M, Horinaka M, et al. Kaempferol sensitizes colon cancer cells to TRAIL-induced apoptosis. Biochem Biophys Res Commun. 2008;375(1):129-133.

14. Choi EJ, Ahn WS. Kaempferol induced the apoptosis via cell cycle arrest in human breast cancer MDA-MB-453 cells. Nutr Res Pract. 2008; 2(4):322-325.

15. Kim SH, Hwang KA, Choi KC. Treatment with kaempferol suppresses breast cancer cell growth caused by estrogen and triclosan in cellular and xenograft breast cancer models. J Nutr Biochem. 2016;28:70-82.

16. Li C, Zhao Y, Yang D, et al. Inhibitory effects of kaempferol on the invasion of human breast carcinoma cells by downregulating the expression and activity of matrix metalloproteinase-9. Biochem Cell Biol. 2015; 93(1):16-27.

17. Lee GA, Choi KC, Hwang KA. Kaempferol, a phytoestrogen, suppressed triclosan-induced epithelial-mesenchymal transition and metastatic-related behaviors of MCF-7 breast cancer cells. Environ Toxicol Pharmacol. 2017;49:48-57.

18. Lu M, Wang T, He M, et al. Tumor suppressor role of miR-3622b-5p in ERBB2-positive cancer. Oncotarget. 2017;8(14):23008-23019.

19. Zhu Y, Tian Y, Du J, et al. Dvl2-dependent activation of Daam1 and RhoA regulates Wnt5a-induced breast cancer cell migration. PLoS One. 2012;7(5):e37823. 
20. Zhu Y, Shen T, Liu J, et al. Rab35 is required for Wnt5a/Dvl2-induced Rac1 activation and cell migration in MCF-7 breast cancer cells. Cell Signal. 2013;25(5):1075-1085.

21. Liu J, Zhang Y, Xu R, et al. PI3K/Akt-dependent phosphorylation of GSK3beta and activation of RhoA regulate Wnt5a-induced gastric cancer cell migration. Cell Signal. 2013;25(2):447-456.

22. Shan X, Wen W, Zhu D, et al. miR 1296-5p inhibits the migration and invasion of gastric cancer cells by repressing ERBB2 expression. PLoS One. 2017;12(1):e0170298.

23. Schettini F, Giuliano M, De Placido S, Arpino G. Nab-paclitaxel for the treatment of triple-negative breast cancer: rationale, clinical data and future perspectives. Cancer Treat Rev. 2016;50:129-141.

24. Kim BW, Lee ER, Min HM, et al. Sustained ERK activation is involved in the kaempferol-induced apoptosis of breast cancer cells and is more evident under 3-D culture condition. Cancer Biol Ther. 2008;7(7): 1080-1089.

25. Diantini A, Subarnas A, Lestari K, et al. Kaempferol-3-O-rhamnoside isolated from the leaves of schima wallichii Korth. inhibits MCF-7 breast cancer cell proliferation through activation of the caspase cascade pathway. Oncol Lett. 2012;3(5):1069-1072.
26. Ahmed S, Sami A, Xiang J. HER2-directed therapy: current treatment options for HER2-positive breast cancer. Breast Cancer. 2015;22(2): 101-116.

27. Devi KP, Malar DS, Nabavi SF, et al. Kaempferol and inflammation: from chemistry to medicine. Pharmacol Res. 2015;99:1-10.

28. Limtrakul P, Khantamat O, Pintha K. Inhibition of P-glycoprotein function and expression by kaempferol and quercetin. J Chemother. 2005; 17(1):86-95.

29. Sharma V, Joseph C, Ghosh S, Agarwal A, Mishra MK, Sen E. Kaempferol induces apoptosis in glioblastoma cells through oxidative stress. Mol Cancer Ther. 2007;6(9):2544-2553.

30. Qin Y, Cui W, Yang X, Tong B. Kaempferol inhibits the growth and metastasis of cholangiocarcinoma in vitro and in vivo. Acta Biochim Biophys Sin (Shanghai). 2016;48(3):238-245.

31. Song H, Bao J, Wei Y, et al. Kaempferol inhibits gastric cancer tumor growth: an in vitro and in vivo study. Oncol Rep. 2015;33(2): $868-874$.

\section{Publish your work in this journal}

OncoTargets and Therapy is an international, peer-reviewed, open access journal focusing on the pathological basis of all cancers, potential targets for therapy and treatment protocols employed to improve the management of cancer patients. The journal also focuses on the impact of management programs and new therapeutic agents and protocols on

\section{Dovepress}

patient perspectives such as quality of life, adherence and satisfaction. The manuscript management system is completely online and includes a very quick and fair peer-review system, which is all easy to use. Visit http://www.dovepress.com/testimonials.php to read real quotes from published authors. 